\title{
Characterization of the Bombyx mori homolog of islet homeostasis protein (IHoP) cDNA: BmIHoP controls insulin secretion from the $\beta$-cells.
}

\author{
Hyojung Shin ${ }^{1}$, Kisang Kwon ${ }^{2}$, Ji-Young Choi ${ }^{3}$, O-Yu Kwon', Seung-Whan Kim ${ }^{4}$ * \\ ${ }^{1}$ Departments of Medical Science and Anatomy and Cell Biology, College of Medicine, Chungnam National University, \\ Daejeon, Korea \\ ${ }^{2}$ Department of Biomedical Laboratory Science, College of Health and Welfare, Kyungwoon University, Gumi, Korea \\ ${ }^{3}$ Applied Entomology Division, National Academy of Agricultural Science, RDA, Wanju, Korea \\ ${ }^{4}$ Department of Emergency Medicine, College of Medicine, Chungnam National University, Daejeon, Korea
}

\begin{abstract}
Islet homeostasis protein (IHoP) was firstly characterized as a novel protein that regulates glucagon synthesis in alpha-cells of pancreatic islet. Here, we report a cDNA designed $B m I H o P$ encoding an IHop homolog cloned from the fat body of the silkworm, Bombyx mori, using conserved domain homology search method. The resultant $B m I H o P$ translated into a protein that encodes 693 amino acids, with a theoretical isoelectric point of 5.15 and a molecular weight of $77.5 \mathrm{kDa}$. Homology analysis revealed that $B m I H o P$ has higher similarity at the amino acid level with those of other species, 52\% identity with $\boldsymbol{H}$. sapiens, $52 \%$ with $M$. musculus, $51 \%$ with $D$. rerio, and $73 \%$ with $D$. melanogaster. The expression of $B m I H o P$ was detected in all tissues tested with 4 fold higher expression in the midgut, Malpighian, ovary and testis, and stronger expression in the larval fat body at $5^{\text {th }}-3^{\text {rd }}$ instar. The overexpression of $\mathrm{BmIHoP}$ in the $\beta$-cells reduced insulin secretion, which is not associated with SOX17 regulation. To the best of our knowledge, this is the first report of $B$. mori IHoP cDNA, BmIHoP, and its associated role in controlling insulin secretion from the $\beta$-cells.
\end{abstract}

Keywords: Silkworm, Bombyx mori, Islet homeostasis protein, cDNA, Insulin.

Accepted on June 29, 2018

\section{Introduction}

The pancreatic islets are small islands composed of 5 different types of cells that produce peptide hormones including glucagon by alpha cells ( $\alpha$-cells), insulin by beta cells ( $\beta$-cells), somatostatin by delta cells ( $\delta$-cells), pancreatic polypeptide by gamma cells (PP-cells), and ghrelin also known as lenomorelin by epsilon cells ( $\varepsilon$-cells) [1-3]. The synthesized hormones are secreted directly into the bloodstream for the homeostasis of blood glucose, fatty acids, triglycerides, and amino acids [4]. Especially, maintaining homeostasis of blood glucose level is regulated by two kinds of hormones, insulin, and glucagon. It is important to maintain the balance between insulin and glucagon in blood, and the involved mechanism is described in brief as follows: when blood glucose level is high, stimulation of pancreas takes place with the subsequent release of insulin from $\beta$-cells. In the case of glucagon, the hormone is released from $\alpha$-cells of the pancreas, when blood glucose levels are low [5,6].

The liver is the main target organ of glucagon, which controls glucose secretion from the stored liver glucose. Glucagon is biosynthesized from the cleavage of proglucagon by proprotein convertase 2 in pancreatic islet $\alpha$-cells [7,8]. While secretion of glucagon is stimulated by hypoglycemia, epinephrine, arginine, alanine, alanine transaminase, acetylcholine, and cholecystokinin, glucagon secretion is inhibited by somatostatin, insulin, PPAR $\gamma /$ retinoid $\mathrm{X}$ receptor heterodimer, increased free fatty acids, and urea production [9-11].

Although extensive research has been carried out with emphasis on type-1 diabetes, the underlying acute mechanisms and pathogenesis are not completely understood. Further research is required to gain deeper insight into the diagnosis and prevention of type- 1 diabetes. Recently, a study reported that a gene co-localized with $\alpha$-cells and encoding islet homeostasis protein (IHoP) produces glucagon from the pancreatic Langerhans islets. The authors demonstrated that IHoP strongly associates with glucagon synthesis, which exhibits its effects on insulin regulation. Inhibition of IHoP expression resulted in the loss of glucagon expression, which induced low blood glucose levels and cellular apoptosis [12]. The data suggested that IHoP might be actively involved in maintaining pancreas homeostasis through the regulation of $\alpha$ cells and $\beta$-cells. 
The silkworm is the larva of the domesticated silk moth, Bombyx mori, which is nourished for the production of raw silk from past hundreds of years in Asian countries such as Korea, China, Japan, and India [13]. Also, silkworm pupae are used as both food and drugs. Especially in Korea, it is used as an anti-diabetic supplements and good source of protein known as 'BeonDeGi'. Some studies demonstrated the effectiveness of silkworm in the treatment of diabetes at the experimental level [14-16]. Henceforth, we can consider that $B m I H o P$ as a supplements or ingredients may play few important roles in maintaining pancreas homeostasis in the mammalian. To be practically used, an oral toluene method that avoids digestion in the stomach and gut into peptides should be considered [17]. In the present work, we have isolated a cDNA encoding BmIHoP from silkworm fat body and characterized by sequence analysis, expression patterns of tissues and developmental stages; comparative evolutionary analyses already reported homologous genes. Also, we demonstrate that BmIHoP expression in the $\beta$-cells reduced insulin secretion in which SOX17 expression was not related and induced apoptosis [18].

\section{Materials and Methods}

\section{Cloning of BmIHoP cDNA}

Total RNA was extracted from the fat body of B. mori (Jam123 $\times$ Jam124) using Trizol reagent (Invitrogen, Carlsbad, CA, USA) and was further treated with RNase-free DNase-I to remove any potential genomic DNA contamination. Candidate DNA fragments were amplified and subsequently confirmed via direct DNA sequencing. The BLAST search was used for the gene identification. PCR was performed using the primers designed with Primer3 (http://simgene.com/Primer3) based on 'Conserved Domain Databases' (NCBI, National Center for Biotechnology Information) and 'Motif Databases' (GenomeNet, Institute for Chemical Research, Kyoto University, Japan), and the number of PCR cycles was optimized in order to stay within the linear range of amplification. The primers used were as follows: $\mathrm{BmIHoP}$ FOR (5'-TTA GGA TCC ATG GCA GAC AAT TCA-3') and BmIHoP-REV (5'-TTA AAG CTT CTA ATT AAT CGA CTC-3'). The resulting PCR product was sub-cloned in the TOPO TA Cloning ${ }^{\circledR}$ plasmid (Invitrogen) and sequenced to confirm specificity. Several clones were finally sequenced to confirm of some mutations may occur by the DNA polymerase in the cloned cDNAs. All other chemicals and drugs were purchased from Sigma Chemical (St. Louis, MO).

\section{Characterization of BmIHoP}

An open reading frame (ORF), molecular weight, and theoretical isoelectric point were estimated by ExPASy (http:// www.expasy.org). The multiple protein sequence alignments and phylogenetic evolutionary analyses were generated using NCBI (http://www.ncbi.nlm.nih.gov/).

\section{Gene expression analysis by RT-PCR}

Silkworms were reared on an artificial diet at temperatures of $24^{\circ} \mathrm{C}-27^{\circ} \mathrm{C}$ and $70 \%-90 \%$ humidity. Samples of total RNA were prepared from acutely dissected tissues from a silkworm, larval fat body on each stage, and Bm5 cells using Trizol and quantified by measuring absorbance at $260 \mathrm{~nm}$. Typically, $2 \mathrm{mg}$ of total RNA was reverse transcribed using random primers (GE Healthcare, Piscataway, NJ, USA) and mMLV reverse transcriptase (Invitrogen). RT-PCR using the forward primer FOR (5'-G ACT GAC AAT GGC AGA CAA TTC-3') and REV (5'-CCG TCT AAT TAA TCG ACT CCT CAG-3') for $\mathrm{BmIHoP}$ fragment was performed for 30 cycles $\left(94^{\circ} \mathrm{C}\right.$ for $30 \mathrm{~s}$; $58^{\circ} \mathrm{C}$ for $30 \mathrm{~s}$; and $72^{\circ} \mathrm{C}$ for $1 \mathrm{~min}$ (only $10 \mathrm{~min}$ in the final cycle)) with Taq DNA polymerase. A single band of $\mathrm{BmIHoP}$ amplicon (382 bp) was detected in RT-PCR.

\section{Mammalian expression vector construction}

The purified PCR product encoding $\mathrm{BmIHoP}$ was ligated at multiple cloning site of pCMV-Taq $2 \mathrm{~B}$ vector (Addgene, Inc., Cambridge, MA, USA) with BamHI and Hind III. The accuracy of the ORF was further verified by sequencing. Transfections were performed with Fugene HD transfection reagent (Promega) with $2 \mu \mathrm{g}$ of reporter plasmids per Ins 1 cellculture dish according to the manufacturer's instructions.

\section{Western blotting}

Immunoblotting was performed according to the standard procedure secreted insulin from the Ins1 cell transfected by pCMV-Taq 2B-BmIHoP was collected and lysed by the addition of SDS sample buffer $(62.5 \mathrm{mM}$ Tris- $\mathrm{HCl} \mathrm{pH} 6.8,6 \%$ $(\mathrm{w} / \mathrm{v}) \mathrm{SDS}, 30 \%$ glycerol, $125 \mathrm{mM}$ DTT, $0.03 \%(\mathrm{w} / \mathrm{v})$ bromphenol blue) and separated by SDS-PAGE. The proteins were transferred to a nitrocellulose membrane, and the membrane was respectively incubated with the rabbit antipolyclonal insulin antibody (Santa Cruz Biotechnology, Inc., Dallas, TX, USA), overnight at $4^{\circ} \mathrm{C}$. The blots were developed using an enhanced chemiluminescence Western blotting detection system kit (Amersham, Uppsala, Sweden).

\section{Results and Discussion}

Diabetes is one of the most common life-related metabolic diseases, and its incidence is gradually increasing by every year. Korea has also experienced a gradual increase in the incidence rate of diabetes from an estimated less than $1 \%$ in 1970 to an estimated $8 \%-10 \%$ in 2000 [19]. Chronic serious complications in the diabetic patients occur as diseases of retina, kidney, peripheral neuron, and peripheral blood vessels [20]. Although there exist various chemicals for improving insulin resistance clinically, such long-term treatments are associated with side effects such as hypoglycemia, obesity, and other diabetes complications [21]. Henceforth, there exists a need for the development of novel anti-diabetic drugs from the natural substances including plants, organisms, and insects. Since ages, the silkworm, B. mori, has been used in the form of 
medicine in Korea, especially for the treatment of diabetic patients.

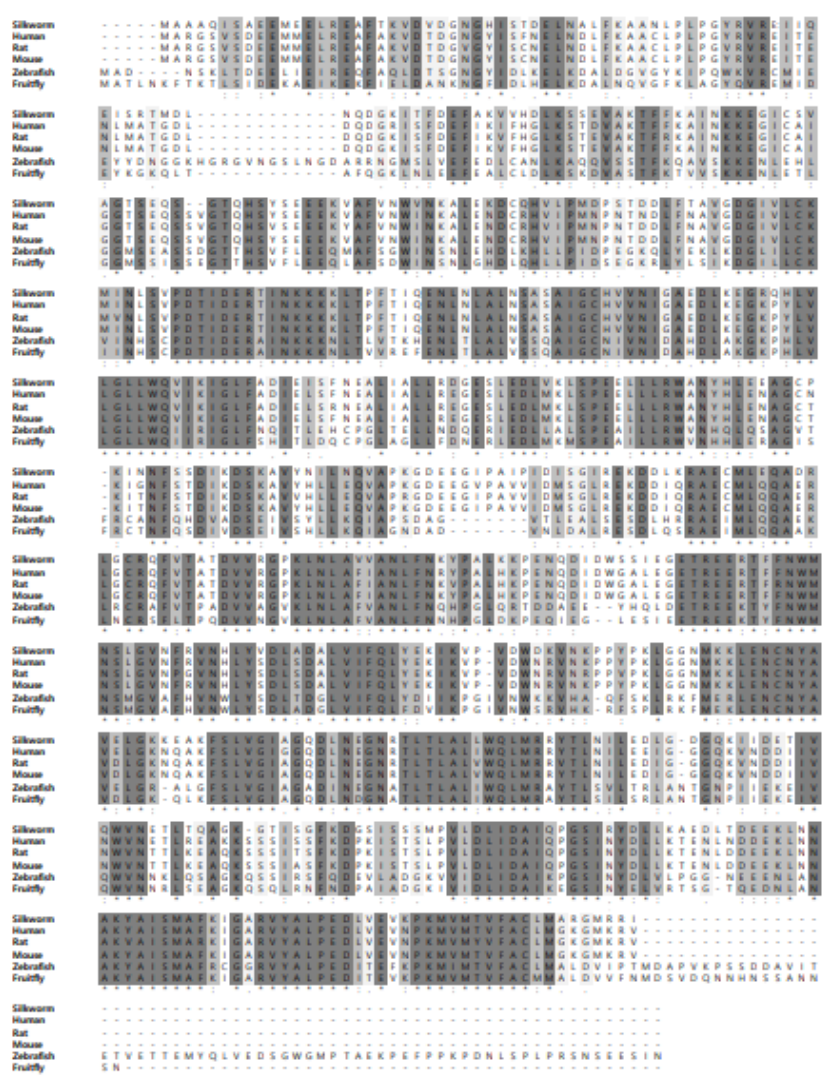

Figure 1. Amino acid sequence alignment of BmIHoP in five different species. A cDNA encoding BmIHoP was successfully cloned through RT-PCR. An alignment of the deduced protein sequence of BmIHoP with other species. Identical amino acid residues in this alignment are indicated by stars. Highly conserved regions of amino acid residues are indicated by points and colons. The deleted positions in the amino acid residues are indicated by dashes. The GenBank accession numbers are as follows, Silkworm (B. mori, KX893487), Human (H. sapiens, NM_002298.4), Rat (R. norvegicus (NM_001012044.1), Mouse (M. musculus, NM_008879.4), Zebrafish (D. rerio, NM_131320.1), and Fruitfly ${ }^{-}$(D. melanogaster, NM_078661.3).

In the present study, we concentrated on the islet homeostasis protein (IHoP), which is firstly reported as a novel factor that regulates glucagon synthesis in the pancreatic alpha-cells. Moreover, we isolated a cDNA encoding $B$. mori IHoP (BmIHoP, GenBank Accession No. KX893487) and described molecular characterization of $\mathrm{BmIHoP}$ and its differential gene expression in different tissues and at different developmental stages. A complete cDNA of $B m I H o P$ shows a typical polyadenylation (AATAAA) signal at the end of 3'-terminal region and an open reading frame (ORF) of a putative protein consisting of 693 amino acids with a theoretical isoelectric point of 5.15 and a molecular weight of $77.5 \mathrm{kDa}$ estimated by ExPASy. As shown in Figure 1, amino acid sequence of $\mathrm{BmIHoP}$ was analyzed based on reported homologues using Basic Local Alignment Search Tool (BLAST) of the NCBI. The amino acid residues of $\mathrm{BmIHoP}$ showed $52 \%$ identity with Homo sapiens, 52\% with Mus musculus, 51\% with Danio rerio, and $73 \%$ with Drosophila melanogaster. The result indicated that $\mathrm{BmIHoP}$ had been relatively well preserved beyond the species with at least $50 \%$ similarity, suggesting that despite little research on its biological function, $\mathrm{BmIHoP}$ is well demonstrated to play a vital role in the pancreatic alphacells.

The phylogenetic tree was generated based on amino acid substitution using to date reported IHoP homologues. The overall tree topology showed that $B m I H o P$ of Lepidoptera $(B$. $m o r i)$ is evolutionarily more closely related to the mouse ( $M$. musculus) IHoP homologue than Diptera (D. melanogaster) IHoP homologue. It is interesting to note that $\mathrm{BmIHoP}$ shows a sister relationship with those of $M$. musculus IHoP homologue; however, rat $(R$. norvegicus) IHoP homologue shows evolutionary distance with $\mathrm{BmIHoP}$ (Figure 2). The information about their topology can greatly reduce experimental difficulties and lead to significant findings concerning their biological functions. Using ExPASy program, we have characterized $\mathrm{BmIHoP}$ on cellular localization and structural topology based on the amino acid sequences. Protein, $\mathrm{BmIHoP}$, is located in the cytosol (estimated about $69 \%$ ) and not in the transmembrane.

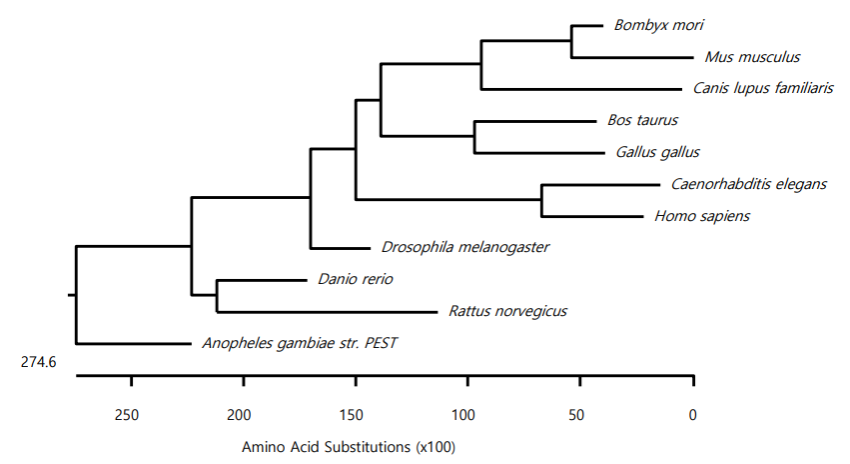

Figure 2. A molecular phylogenetic tree of BmIHoP gene. Phylogenetic tree based on the BmIHoP of various species constructed by the NCBI program. The length of the section indicates the relative distances between the sequences. The GenBank accession numbers are as follows, B. mori (KX893487), M. musculus (NM_008879.4), C. familiaris (XP_005633941.1), B. taurus (NM_001034720.2), G. gallus (NM_001008440.1), C. elegans (NM_067660.4), H. sapiens (NM_002298.4), D. melanogaster (NM 078661.3), $\quad D . \quad$ rerio $(N M-131320.1), \quad R . \quad$ norvegicus (NM_001012044.1), and A. gambiae str. PEST (XM_309626.4).

The mRNA expression of $B m I H o P$ was analyzed in the various tissues of $B$. mori using reverse transcriptional (RT)-PCR (Figures 3A-3C). The result showed that mRNA of $\mathrm{BmIHoP}$ is ubiquitously expressed in all the tested tissues, especially 4folds higher expression was detected in the midgut, Malpighian, ovary and testis as compared to expression in the skin (Figure 3A). The ubiquitous expression of $\mathrm{BmIHoP}$ suggested that $B m I H o P$ is one of the major factors in the biological processes such as glucose homeostasis and insulinglucagon biosynthesis. To understand the developmental expression of $\mathrm{BmIHoP}$, we tested the expression levels in the egg, larva, pupa, and adult stages. It was observed that BmIHoP mRNA expression remained constant (almost at the 
same level) without undergoing any drastic changes during developmental stages (Figure 3B). On the other hand, overall, no dramatic changes in $\mathrm{BmIHoP}$ gene expression in the instar were detected; however, relatively high expression of about 1.5 -folds in the $5^{\text {th }}-3^{\text {rd }}$ instar was observed (Figure 3C).
Although the details of the molecular mechanism of $\mathrm{BmIHoP}$ are not currently understood, the result is that $\mathrm{BmIHoP}$ gene expression shows sever in the various tissues rather than at development stages.

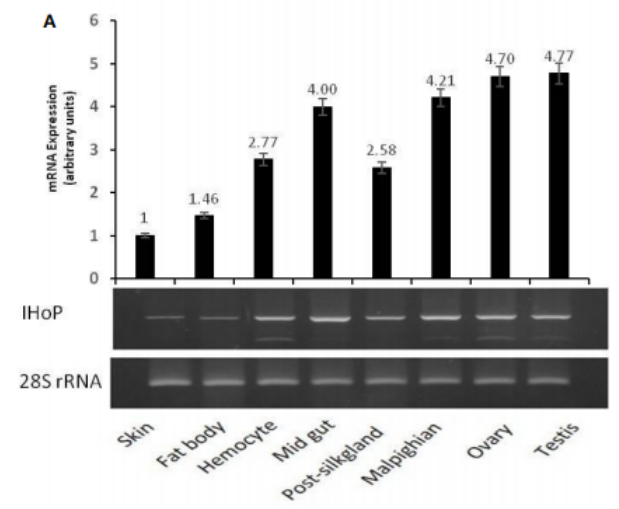

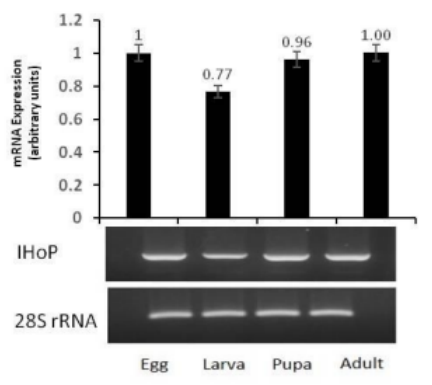

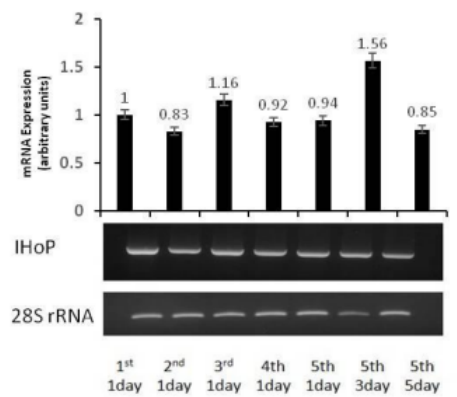

Figure 3. The expression level of BmIHoP mRNA in various tissues and developmental stages. (A) Various tissues were obtained skin, fat body, hemolymph, mid gut, post-silkgland, Malpighian, ovay and testis, respectively; (B) Samples were obtained at different developmental stages ranging from the stage of an egg to adult; (C) Samples obtained from $1^{\text {st }}$ to $5^{\text {th }}$ instars. The expression of $28 S$ rRNA was used as an internal control for assessing the expression level of BmIHoP. RT-PCR was 3 times measured and those average values are represented by bar-graphs.

$\mathrm{OH}$ et al. reported that IHoP is expressed in the pancreatic islet $\alpha$-cells synthesizing glucagon, and it controls the cellular proliferation of $\beta$-cells under high glucose conditions [12]. It is suggested that IHoP could be a new functional protein for the maintenance of pancreatic homeostasis, and one of the new targets for type 1 diabetes treatment. We have constructed a cell line using Ins1 synthesizing insulin in which $\mathrm{BmIHoP}$ gene is over-expressed $\left(\mathrm{BmIHoP}{ }^{\text {over }}\right)$ to understand the association between $B m I H o P$ and insulin secretion. It was observed that $\mathrm{BmIHoP}$ expression is greatly reduced (approximately half) insulin secretion from the $\beta$-cells (Figure 4A). Recently, it has been demonstrated that Sox17 (SRY-Box 17) is a key transcriptional regulator of the insulin trafficking and responsible for secretion of a hormone in the normal and

A

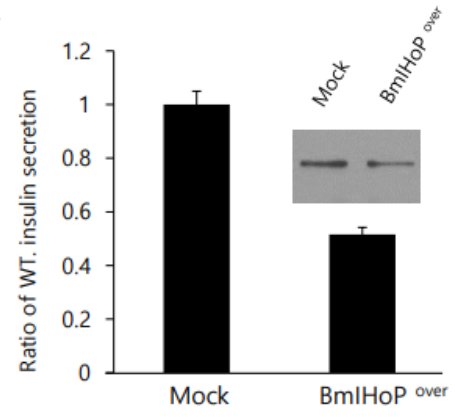

pathologic mouse $\beta$-cells [18]. In this study, our data suggests that Sox17 expression is indirectly associated with the functional $\mathrm{BmIHoP}$ (Figure 4B) for the insulin secretion in the $\beta$-cells. A gene family of Bcl-2 encoding protein that can either suppress (e.g. Bcl-2 and Bcl-XC) or promote (e.g. Bid and Bak) apoptosis; It is also know that intracellular p53 protein promote apoptosis. The $\beta$-cells overexpressing $\mathrm{BmIHoP}$ induces only Bid expression in the MOCK, neither p53 nor $\mathrm{Bcl}-2$. Eventually, the result can say that $\mathrm{BmIHoP}$ functionally induces apoptosis in the $\beta$-cells (Figure 4C). In future, more studies are necessitated to reveal the underlying mechanisms of insulin secretory defection and apoptosis induction by the $\mathrm{BmIHoP}$ in the $\beta$-cells.
B

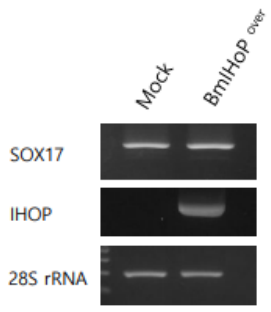

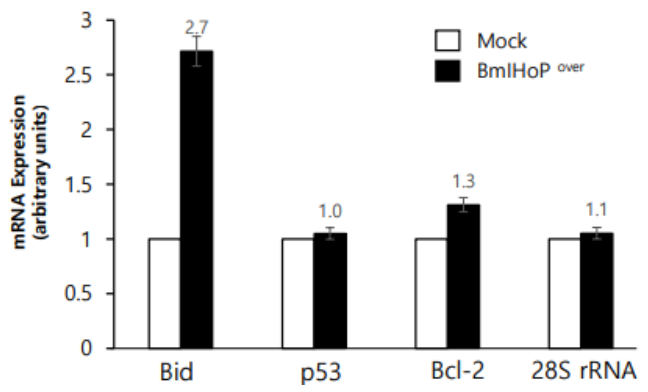

Figure 4. Forced BmIHoP expression in the $\beta$-cells. (A) Insulin secretion in the BmIHoP overexpressed $\beta$-cell by Western blotting; (B) Both the expression of SOX17; (C) Induction of apoptosis by RT-PCR. PPIA expression was used as an internal control for assessing the expression level of BmIHoP. Bar graphs represent the average of three RT-PCR values.

In this study, IHoP homologue $(B m I H o P)$ was isolated from $B$. mori; its gene expression was detected widely in all tissues revealing that the expression was regulated specifically, but not developmentally. Phylogenetic analysis revealed that $\mathrm{BmIHoP}$ shows a sister relationship with $M$. musculus IHoP homologue.
Overexpression of $\mathrm{BmIHoP}$ in the $\beta$-cells reduced insulin secretion and induced apoptosis. Overall, it is hypothesized that the present data on $\mathrm{BmIHoP}$ will provide more information on understanding pancreatic homeostasis. Eventually, it is 
secretion from the $\beta$-cells

proposed that $B m I H o P$ can potentially be applied for treatment of diet-induced obesity in the future.

\section{Acknowledgement}

This study was supported by a grant from the Agenda program (PJ010864), Rural Development Administration, Republic of Korea.

\section{References}

1. Brereton MF, Vergari E, Zhang Q, Clark A. Alpha, delta and PP-cells: Are they the architectural cornerstones of islet structure and co-ordination? J Histochem Cytochem 2015; 63: 575-591.

2. Otter S, Lammert E. Exciting times for pancreatic islets: Glutamate signaling in endocrine cells. Trends Endocrinol Metab 2016; 27: 177-188.

3. Weir GC. Islet-cell biology in 2015: Understanding secretion, ageing and death in $\beta$ cells. Nat Rev Endocrinol 2016; 12: 72-74.

4. Saltier AR. Insulin signaling in the control of glucose and lipid homeostasis. Handb Exp Pharmacol 2016; 233: 51-71.

5. Bankir L, Bouby N, Blondeau B, Crambert G. Glucagon actions on the kidney revisited: possible role in potassium homeostasis. Am J Physiol Renal Physiol 2016; 311: 469-486.

6. Röder PV, Wong X, Hong W, Han W. Molecular regulation of insulin granule biogenesis and exocytosis. Biochem J 2016; 473: 2737-2756.

7. Miller RA, Birmbaum MJ. Glucagon: acute actions on hepatic metabolism. Diabetologia 2016; 59: 1376-1381.

8. Wang XC, Gusdon AM, Liu H, Qu S. Effects of glucagonlike peptide-1 receptor agonists on non-alcoholic fatty liver disease and inflammation. World J Gastroenterol 2014; 20: 14821-14830.

9. Blair M. Diabetes mellitus review. Urol Nurs 2016; 36: 27-36.

10. Sandoval DA, D' Alessio DA. Physiology of proglucagon peptides: role of glucagon and GLP-1 in health and disease. Physiol Rev 2015; 95: 513-548.

11. Tsuda T. Possible abilities of dietary factors to prevent and treat diabetes via the stimulation of glucagon-like peptide-1 secretion. Mol Nutr Food Res 2015; 59: 1264-1273.

12. Oh SH, Darwiche H, Cho JH, Shupe T, Petersen BE. Characterization of a novel functional protein in the pancreatic islet: islet homeostasis protein regulation of glucagon synthesis in $\alpha$ cells. Pancreas 2012; 41: 22-30.

13. Zabelina V, Klymenko V, Tamura T, Doroshenko K, Liang $\mathrm{H}$, Sezutsu H, Sehnal F. Genome engineering and parthenocloning in the silkworm, Bombyx mori. J Biosci 2015; 40: 645-655.

14. Do SG, Park JH, Nam H, Kim JB, Lee J, Oh YS, Suh JG. Silk fibroin hydrolysate exerts an anti-diabetic effect by increasing pancreatic $\beta$ cell mass in $\mathrm{C} 57 \mathrm{BL} / \mathrm{KsJ}-\mathrm{db} / \mathrm{db}$ mice. J Vet Sci 2012; 13: 339-344.

15. Heo HS, Choi JH, Oh JJ, Lee WJ, Kim SS, Lee DH, Lee HK, Song SW, Kim KH, Choi YK, Ryu KS, Kang BH. Evaluation of general toxicity and genotoxicity of the silkworm extract powder. Toxicol Res 2013; 29: 263-278.

16. Zhang Y, Wang N, Wang W, Wang J, Zhu Z, Li X. Molecular mechanisms of novel peptides from silkworm pupae that inhibit $\alpha$-glucosidase. Peptides 2016; 76: 45-50.

17. Weiner HL, da Cunha AP, Quintana F, Wu $\mathrm{H}$. Oral tolerance. Immunol Rev 2011; 241: 241-259.

18. Jonatan D, Spence JR, Method AM, Kofron M, Sinagoga K, Haataja L, Arvan P, Deutsch GH, Wells JM. Sox17 regulates insulin secretion in the normal and pathologic mouse $\beta$ cell. PLoS One 2014; 9: e104675.

19. Ha KH, Kim DJ. Current status of managing diabetes mellitus in Korea. Korean J Intern Med 2016; 31: 845-850.

20. Gomes MB, Negrato CA, Almeida A, de Leon AP. Does parity worsen diabetes-related chronic complications in women with type 1 diabetes? World J Diabetes 2016; 7: 252-259.

21. Staiger H, Schaeffeler E, Schwab M, Häring HU. Pharmacogenetics: Implications for modern type 2 diabetes therapy. Rev Diabet Stud 2015; 12: 363-376.

\section{*Correspondence to}

Seung-Whan Kim

Department of Emergency Medicine

College of Medicine

Chungnam National University

Daejeon

Korea 